\title{
Optimal power allocation and power constraint in OFDM-Based cognitive radio systems
}

\author{
Nguyen Van Vinh ${ }^{1,2}$, Pham Ngoc Thang ${ }^{2}$ \\ ${ }^{1}$ School of Information Engineering, Zhengzhou University, China \\ ${ }^{2}$ Faculty of Electronic and Electrical Engineering, Hung Yen University of Technology and Education, Hung Yen, Vietnam \\ Email address: \\ vinhnv.edu@gmail.com (N. V. Vinh), phamngocthangutehy@gmail.com (P. N. Thang)
}

\section{To cite this article:}

Nguyen Van Vinh, Pham Ngoc Thang. Optimal Power Allocation and Power Constraint in OFDM-Based Cognitive Radio Systems. American Journal of Networks and Communications. Vol. 3, No. 4, 2014, pp. 49-55. doi: 10.11648/j.ajnc.20140304.11

\begin{abstract}
Cognitive Radio (CR), which aims to improve spectrum utilization by accessing the spectrum originally licensed to a primary system, is a promising concept. In cognitive radio system based on orthogonal frequency division multiplexing (OFDM), the optimized algorithms for sub-carrier power allocation share the problems of complex iterative calculation and difficult realization. In this case, a sub-optimal power allocation algorithm based on power function distribution is proposed, to allocate power of in-band subcarrier of cognitive user according to the numerical characteristics of the power function by using a convex optimization numerical method under linear constraint. This algorithm has the advantages of fast calculation speed and easy realization, and reduces the interference for the authorized user brought by the power leakage of the in-band subcarrier of cognitive user appears in the out-of-band. MALAB simulation results show that the proposed scheme maximizes the in-band channel capacity of the cognitive user under the interference threshold of the authorized user and the comparison model the optimal relationship between the limited and unlimited transmit power capacity. Meanwhile, the maximum transmission rate of the existing power allocation scheme is increased within the same condition.
\end{abstract}

Keywords: Orthogonal Frequency-Division Multiplexing (OFDM), Cognitive Radio (CR), Power Allocation, Linear Water-Filling, Convex Optimization

\section{Introduction}

The available resource of wireless spectrum is becoming scarcer, and it seriously impedes the exploitation and application of new technique due to the explosive growth of wireless communication. Cognitive radio [1] has been widely used as an effective method of improving the wireless spectrum utilization. It can perceive and detect the dynamic changes of idle wireless spectrum. CR is the most effective way of solving the problem of the spectrum scarcity [2]. The cognitive users $(\mathrm{CU})$ can communicate with each other by automatically searching and utilizing the idle spectrum based on not affecting the normal communications of the license users (LU).

The orthogonal frequency division multiplexing technology can highly improve the performance of cellular system by utilizing the characteristic of multiuser diversity to distribute the sub-channel, bit and power effectively. It is regarded as an ideal alternative technology of realizing CR system [3]. CU can neatly fill the idle spectrum left by LU. Additionally, the module of fast Fourier transform (FFT) in the receiver can be used for spectrum perception meanwhile.

Based on above discussions, the OFDM modulation technique is both used in CU and LU, Weiss T. A. et al. [4] studied the mutual interference caused by non-orthogonality between $\mathrm{CU}$ and LU based on the convex optimization theory. G. Bansal et al. [5] studied the resource allocation plan base on OFDM in CR network. Moreover, according to the traditional power allocation scheme (such as water-filling algorithm), more power should be distributed to the sub carrier with higher quality channel. Wyglinski [6] proposed an unequal bit loading algorithm for a non-contiguous OFDM-Based CR system. In fact such an interference limited scenario limits the transmit power as well as the achievable transmission rate of CR users.

In this paper, the design problem for maximizing the transmission rate of the CR user, therefore, was proposed. The present study successfully set up an optimization problem of maximizing the $\mathrm{CU}$ channel capacity by using Lagrange multiplier method. The optimal solution of this mathematical problem was also obtained. Power allocating on the 
subcarriers is the exponential distribution function with the purpose of optimizing channel capacity at the CU band. We have also compared the present results with results in [7]. This paper sets up an optimization problem of maximizing the $\mathrm{CU}$ channel capacity by using the Lagrange multiplier method. Using this method the optimal solution can be obtained.

The rest of the paper is organized as follows: Section 2 presents the system model, and formulates the studied problem as a convex optimization problem; Section 3 proposed the optimal algorithm to solve the formulated problem, and two suboptimal schemes are also developed; Section 4 provides numerical simulations, and finally concluding remarks are presented in Section 5.

\section{The Dowlink of CR System Model}

According to how the IEEE 802.11a system model [4] utilizes the OFDM modulation technique, the disposition of LU band and idle spectrum are shown in Fig.1.

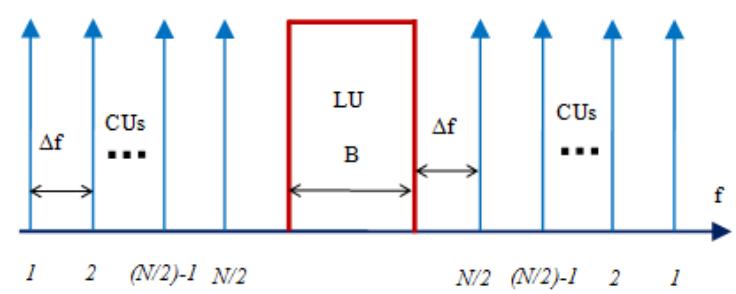

Fig 1. Distribution of license and cognitive users.

Where the LU's bandwidth is $B$, and CUs separate in the two sides of LU. The interval of every subcarrier in the CU band, and the interval between LU band and CU band are both $\Delta f$. The sum of subcarriers in the band of CU is $N$. Because the OFDM modulation mode is used both in CUs and LU (the attenuation characteristic of power spectral density side lobe), the LU and CUs both have interferences on each other.

\subsection{Interference Introduced by Cognitive User's Signal}

According to S. Haykin [1], the power density spectrum of the $i_{\text {th }}$ subcarrier in CR user band can be written as:

$$
\varphi_{i}(f)=P_{i} T_{s}\left(\frac{\sin \pi f T_{s}}{\pi f T_{s}}\right)^{2}
$$

Where $P_{i}$ denotes the transmitting power of subcarrier $i$ in the $\mathrm{CU}$ band, $T_{s}$ denotes the symbol period of OFDM. Therefore, the interference power of signal in subcarrier $i$ in $\mathrm{CU}$ band to $\mathrm{LU}$ is:

$$
I_{i}\left(d_{i}, p_{i}\right)=P_{i} \times T_{s} \int_{d_{i-B / 2}}^{d_{i+B / 2}}\left(\frac{\sin \pi f T_{s}}{\pi f T_{s}}\right)^{2} d f
$$

Which defines:

$$
K_{i}^{l}=T_{s} \int_{d_{i-B / 2}}^{d_{i+B / 2}}\left(\frac{\sin \pi f T_{s}}{\pi f T_{s}}\right)^{2} d f
$$

Therefore, the formula (2) can be changed as:

$$
I_{i}\left(d_{i}, P_{i}\right)=P_{i} \times K_{i}
$$

Where $d_{i}$ denotes the nearest spectrum distance between the subcarrier $i_{\text {th }}$ and LU band in CU band.

\subsection{Interference Introduced by License User's Signal}

The interference power of LU signal after the M-fast Fourier transform (FFT) processing to subcarrier $i$ in CU band is

$$
I_{L U}\left(d_{i}, P_{L U}\right)=T_{s} \int_{d_{i-B / 2}}^{d_{i+B / 2}} \varphi_{L U}(f) d f
$$

Where $\varphi_{\mathrm{LU}}(\mathrm{f})$ denotes the PSD of LU signals; $P_{L U}$ denotes power of LU signals.

\subsection{Channel Throughput Analysis of CUs [7]}

Assuming that each subcarrier goes under frequency flat fading and the instantaneous fading gains are perfectly known at the transmitter. The transmit power adaptively loaded in each CR user's subcarrier. With an ideal coding scheme, the transmission rate at $i_{t h}$ subcarrier, $R_{i}$ for the transmit power, $P_{i}$ and channel fading gain $h_{i}$ is connected via the Shannon capacity formula and is given by:

$$
R_{i}=\log _{2}\left(1+\frac{\left|h_{i}\right|^{2} \times P_{i}}{\Gamma \times \sigma_{i}^{2}}\right)
$$

Where $\sigma_{i}^{2}$ is the sum power of Gaussian noise and the interference caused by PUs on $i_{t h}$ subcarrier. $\Gamma$ is a constant signal-to-noise ratio (SNR) gap, according to [8], $\Gamma$ has a relationship with the required bit error rate (BER):

$$
\Gamma=\frac{\ln (5 B E R)}{1.5}
$$

\section{Optimal Power Allocation}

The optimal power allocation algorithm and two suboptimal power allocation algorithms were investigated. They have less complexity.

$$
\begin{array}{r}
C=\max _{P_{i}} \sum_{i=1}^{N} R_{i}\left(P_{i}, h_{i}\right) \\
\text { s.t. }\left\{\begin{array}{l}
\sum_{i=1}^{N} I_{i}^{1}\left(d_{i}, P_{i}\right) \leq I_{t h}^{1} \\
\sum_{i=1}^{N} I_{i}^{2}\left(d_{i}, P_{i}\right) \leq I_{t h}^{2} \\
P_{i} \geq 0
\end{array}\right.
\end{array}
$$

Where $C$ is CU's channel capacity, $N$ is the total number of subcarrier in $\mathrm{CU}$ band, $R_{i}$ is the transmission rate on $i_{t h}$ subcarrier, and the interference thresholds prescribed by the 
right and left primary bands are equal, e.g. $I_{t h}^{1}=I_{t h}^{2}=I_{t h}$.

\subsection{Optimal Power Allocation Scheme}

For the above optimization problem, although the objective function is a concave function, the constraint condition is linear. Thus the optimization problem is a convex optimization problem. And the convex optimization approach can be used to analyze the optimal power allocation scheme. We use Lagrange multiplier method to construct the Lagrange function:

$$
\begin{gathered}
L\left(P_{i}, \lambda_{1}, \lambda_{2}\right)=\sum_{i=1}^{N} R_{i}\left(P_{i}, h_{i}\right)-\lambda_{1} . \\
\left(\sum_{i=1}^{N} I_{i}^{1}\left(d_{i}, P_{i}\right)-I_{t h}^{1}\right)-\lambda_{2}\left(\sum_{i=1}^{N} I_{i}^{2}\left(d_{i}, P_{i}\right)-I_{t h}^{2}\right)
\end{gathered}
$$

Where $\lambda_{1}$ and $\lambda_{2}$ are the Lagrange constraints, $\lambda_{1}, \lambda_{2} \geq 0$.

Therefore, the formula (10) derivative can be obtained:

$$
\frac{\partial L}{\partial P_{i}}=\frac{1}{\frac{\delta^{2}}{\left|h_{i}\right|^{2}}+P_{i}}-\lambda_{1} \frac{\partial I_{i}^{1}}{\partial P_{i}}-\lambda_{2} \frac{\partial I_{i}^{2}}{\partial P_{i}}
$$

Which defines:

$$
\delta_{i}^{2}=\delta^{2}+I_{L U} ; K_{i}^{l}=\frac{\partial I_{i}^{l}}{\partial P_{i}}=T_{S} \int_{d_{i}-B / 2}^{d_{i}+B / 2}\left(\frac{\sin \pi f T_{S}}{\pi f T_{S}}\right)^{2} d f
$$

The right side of equation (11) is equal to zero. Therefore, the that get $P_{i}$ optimal solution:

$$
P_{i}^{*}=\left(\frac{1}{\lambda_{1} k_{i}^{1}+\lambda_{2} k_{i}^{2}}-\frac{\delta_{i}^{2}}{\left|h_{i}\right|^{2}}\right)^{+}
$$

Where $(\mathrm{x})^{+}=\max (0, \mathrm{x})$ and Lagrange multiplier $\lambda_{1}, \lambda_{2}$ can be formula (13) are obtained:

$$
\left\{\begin{array}{l}
\sum_{i=1}^{N} I_{i}^{1}\left(d_{i}, P_{i}^{*}\right)=I_{t h} \\
\sum_{i=1}^{N} I_{i}^{2}\left(d_{i}, P_{i}^{*}\right)=I_{t h}
\end{array}\right.
$$

From equation (13), we can see that the power is assigned to the $i_{t h}$ carrier of the CU band may appear less than zero. Therefore, here carried out the processing and analysis steps iterative partitioned water filling (IPW) algorithms. After much choice and change of operator until the allocated power on each carrier is non-negative and positive in the $\mathrm{CU}$ band [9].

\subsection{Suboptimal Scheme}

By using the above scheme, we can calculate the optimal power allocation policy that maximizes the transmission capacity of the CR user while keeping the interference introduced to the Pos below the specified threshold. However, the complexity of the optimal scheme is high and hence, in the following section, we propose suboptimal schemes based on heuristics that have lower complexity.

In the scheme optimized power allocation, the number of carriers in the $\mathrm{CU}$ band needs to be adjusted from left to right are $1,2,3, \ldots,(N / 2)-1, N / 2, N / 2,(N / 2)-1, \ldots, 3,2,1$, where $N$ is an even number; Similarly when $N$ is odd. Ref. [7] proposed optimization scheme A and B. The authors proposed schemes $\mathrm{C}$ and $\mathrm{D}$, based on power allocation functions exponential.

\subsubsection{Scheme C}

In this algorithm, determination of the maximum power can be allocated in each subcarrier is different. We divide the total interference on the number of the available subcarriers, and equal interference threshold per subcarrier will be determined. In this way the maximum power that can be allocated to each subcarrier will be:

$$
P_{i}^{C}=P \times i^{1.5}, i=1,2,3, \ldots, N
$$

where $P$ will be determined by the value of $I_{t h}$. In conjunction with equation (13) can be written as

$$
P_{i}^{C^{*}}=\frac{i^{1.5} \times I_{t h}}{\sum_{i=1}^{N} i^{1.5} \times K_{i}}
$$

\subsubsection{Scheme D}

The classic OFDM loading algorithms: uniform power loading and water filling schemes are suboptimal for such a interference limited scenario as they do not have constraint on the interference. Therefore, for a given interference threshold $I_{t h}$, power allocated to the $i_{t h}$ subcarrier with uniform power loading, can easily expressed as:

$$
P_{i}^{D}=P \times i^{2.5}, i=1,2,3, \ldots, N
$$

where $P$ will be determined by the value of $I_{t h}$. In conjunction with equation (13) can be written as

$$
P_{i}^{D^{*}}=\frac{i^{2.5} \times I_{t h}}{\sum_{i=1}^{N} i^{2.5} \times K_{i}}
$$

\section{Simulation Results}

In the process of simulation, we suppose the values of $T_{s}, \Delta f$ and $B$ to be $4 \mu \mathrm{s}, 0.3125 \mathrm{MHz}, 0.3125 \mathrm{MHz}$ respectively. The total number $\mathrm{N}$ of sub carriers in $\mathrm{CU}$ channel is 12 , which means there are six subcarriers in each side of LU channel (It's similar when $\mathrm{N}$ is an odd number). The noise power of every 
subcarrier is in $(1,10) \mu \mathrm{W}$ randomly, the white Gaussian noise in LU channel is $10 \mu \mathrm{W}$, the interference threshold is $2.2 \mathrm{~mW}$, the total transmitting power of CU channel is $1 \mathrm{~W}$, the bit error rate is $10^{-3}$, the results are based on perfect channel state information (CSI) at the transmitter and receiver. The present results are presented as below.

Figure 2 presents the schematic diagram of the power distribution of above four methods [10]. The methods of optimal capacity allocation are expressed by intuition and could be observed on both sides of the $\mathrm{CU}$ band from the diagram LU band.

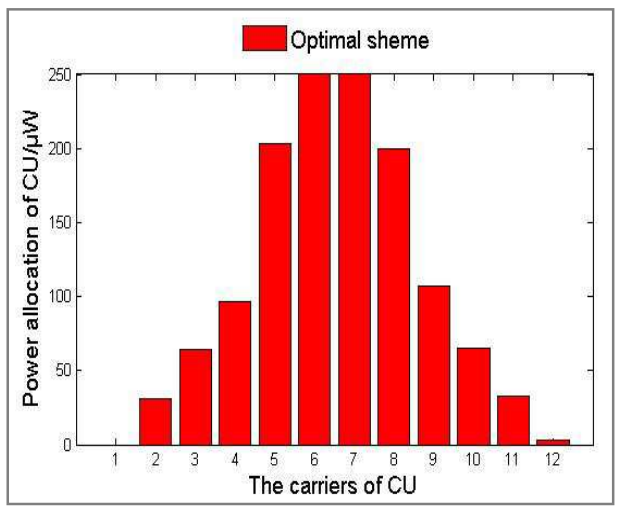

(a) Optimal scheme

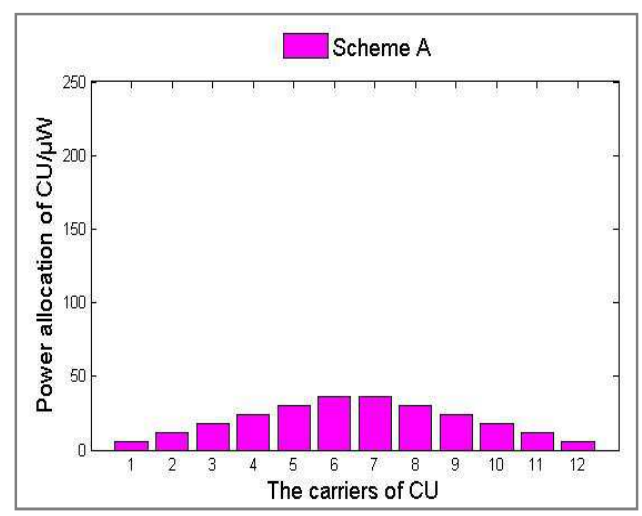

(b) Scheme A

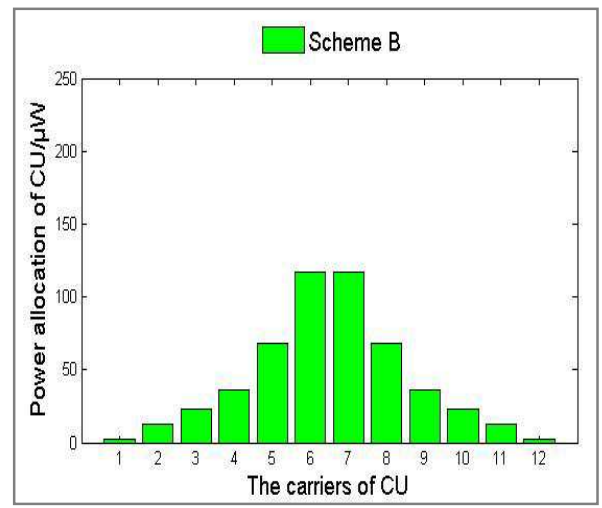

(c) Scheme B

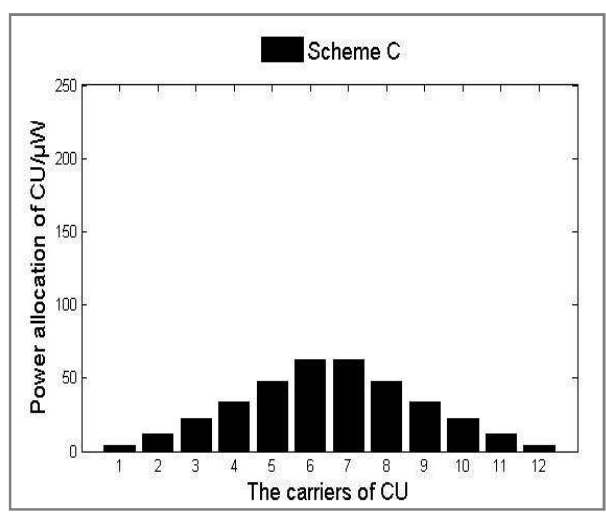

(d) Scheme C

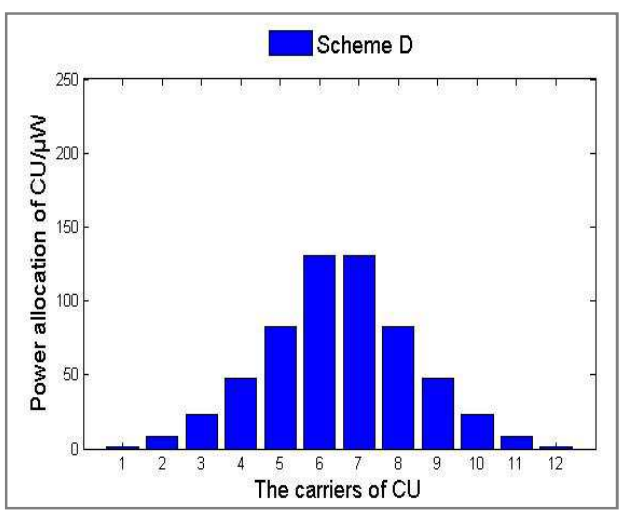

(e) Scheme D

Fig 2. Power distribution histograms of different schemes.

Table 1 represents the power distribution of Figure 2 corresponding to the optimal allocation method. Four methods of allocating sub-optimal capacity are analyzed based on qualitative perspective. Moreover, relationship between the transmit powers of the sub-carrier CU bands is inversely proportional to the distance to the LU band and greater than transmission capacity of the carrier LU band. Therefore, it is easy to calculate the optimum power allocation and easily used in practical applications.

Figure 3 presents one example realization of the channel power (i.e. $\left|h_{i}\right|^{2}$ ) corresponding to the 12 subcarrier of the CU band. These values are $0.3293,0.4261,1.3712,2.1310,2.1909$, $1.7106,0.7362,1.3674,0.8638,1.7524,0.4715$, and 0.5771 $\mathrm{dB} \mu$ respectively. Again, these values are random created within the range $[1,10] \mu \mathrm{W}$.

Figure 4 presents the achievable capacity of CR user versus interference introduced to the primary user band for different schemes under considerations. As we can see from the figure, all capacity performance increases with the increasing interference threshold. In addition, the performance of scheme $\mathrm{D}$ is very closely to the optimal power allocation scheme with lower complexity.

Figure 5 presents the transmit power of the CR user versus the interference introduced to the primary user's band for various scheme under consideration. We can observe that the optimal scheme allows to transmit high power than the other schemes for given interference threshold, as the optimal scheme take judiciously interference into account in its power 
loading policy for a given interference threshold.

In figures 6 and 7, we examine the channel capacity of the CU channels in our proposed suboptimal scheme D in comparison with that in the optimal power allocation scheme, using the same simulation parameters and conditions mentioned in the previous simulation results, except that the exponent, denoted as $x$, takes different values 1.0, 1.5, 2.0, 2.5, 3.0, and 3.5. In particular, figure 6 presents the channel capacity of CUs in our proposed power allocation scheme D with different exponential distributions with different values of the exponent, while figure 7 magnifies figure 6 for the two example ranges of interference thresholds $1.45 \leq \mathrm{I}_{\text {th }} \leq 1.55$ and $2.95 \leq \mathrm{I}_{\mathrm{th}} \leq 3.05$. From figure 6 and 7 , one can see clearly that the proposed scheme $\mathrm{D}$ with the exponent of 3.0 has the best performance for the whole considered range of interference thresholds.

Tab 1. The power values of different options scheme $(\mu \mathrm{W})$.

\begin{tabular}{|c|c|c|c|c|c|c|c|c|c|c|c|c|}
\hline \multirow{2}{*}{ The schemes } & \multicolumn{12}{|c|}{ The values subcarrier } \\
\hline & 1 & 2 & 3 & 4 & 5 & 6 & 7 & 8 & 9 & 10 & 11 & 12 \\
\hline Opt. scheme & $5.1 \mathrm{e}-19$ & 31.43 & 64.41 & 96.91 & 202.87 & 334.15 & 332.27 & 199.31 & 106.76 & 65.31 & 32.44 & 3.34 \\
\hline Scheme A & 6.11 & 12.23 & 18.35 & 24.46 & 30.58 & 36.70 & 36.70 & 30.58 & 24.46 & 18.35 & 12.23 & 6.11 \\
\hline Scheme B & 2.19 & 12.78 & 22.63 & 35.76 & 68.08 & 117.23 & 117.23 & 68.08 & 35.76 & 22.63 & 12.78 & 2.19 \\
\hline Scheme C & 4.22 & 11.95 & 21.96 & 33.81 & 47.25 & 62.12 & 62.12 & 47.25 & 33.81 & 21.96 & 11.95 & 4.22 \\
\hline Scheme D & 1.47 & 8.37 & 23.06 & 47.35 & 82.72 & 130.49 & 130.49 & 82.72 & 47.35 & 23.06 & 8.37 & 1.47 \\
\hline
\end{tabular}

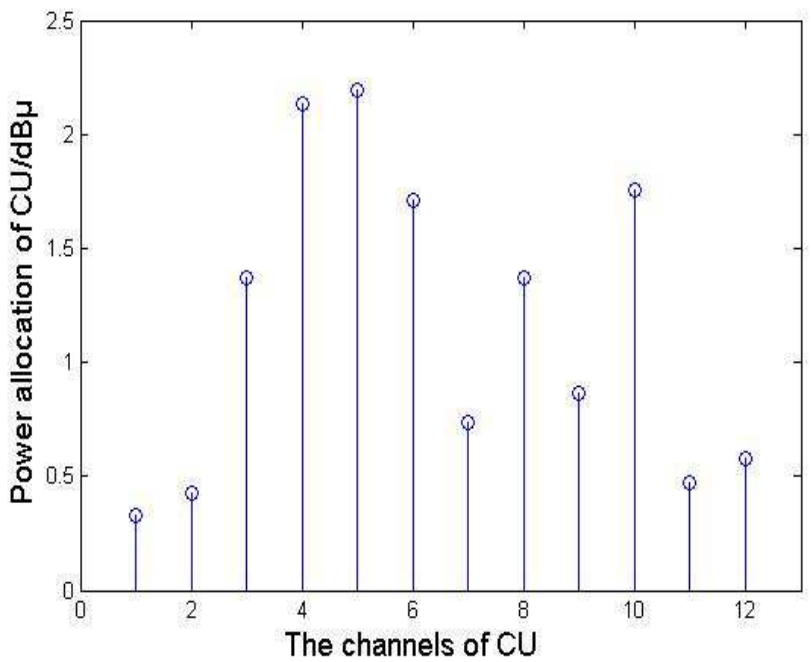

Fig 3. Power allocation on channels of $C U$.

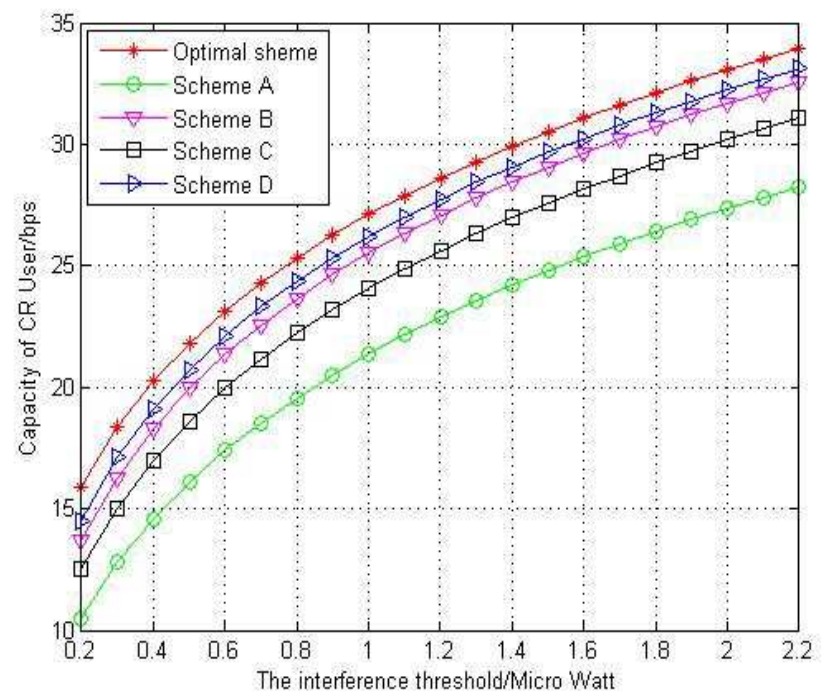

Fig 4. Under different interference threshold of the maximum transmission rate of $C U$.

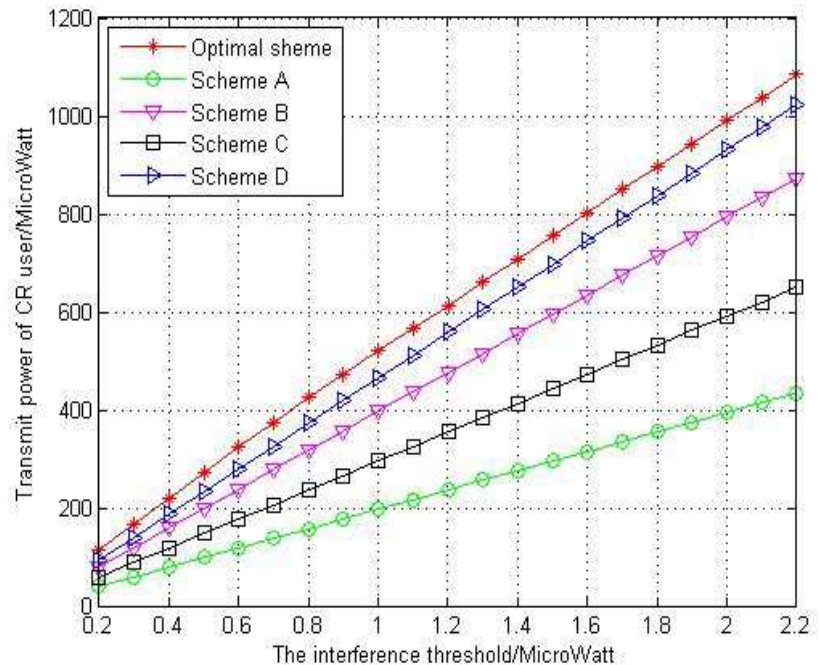

Fig 5. CU under different interference threshold of the maximum transmit power.

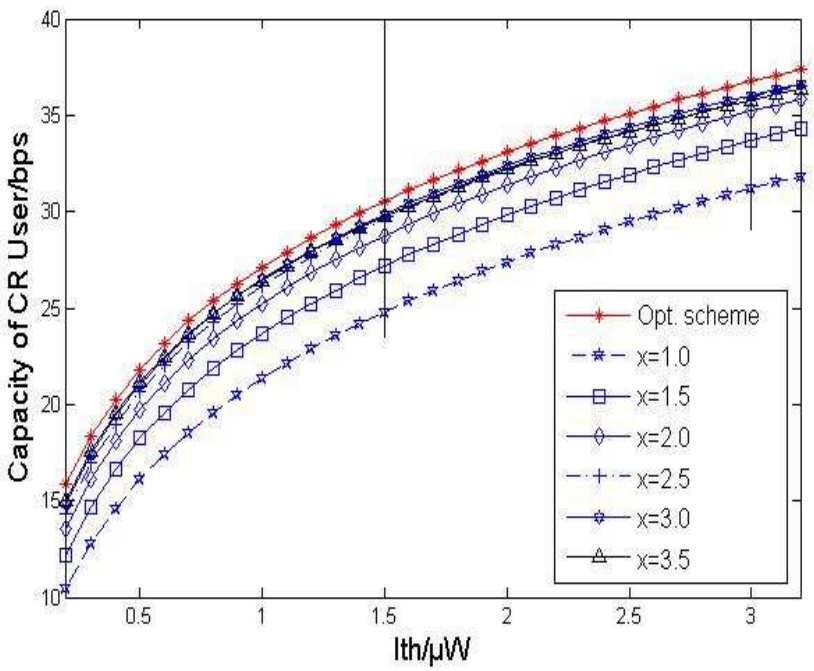

Fig 6. Maximum transmission rate of CUs under different power exponent values. 

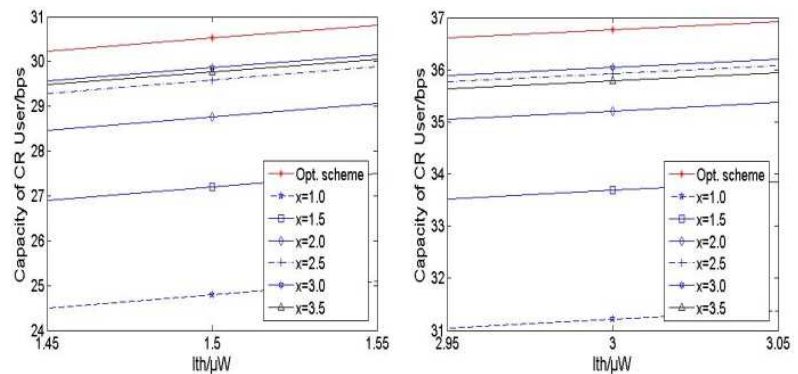

Fig 7. Magnification of Fig. 6 in the interference threshold ranges $1.45 \leq I_{\text {th }} \leq$ 1.55 and $2.95 \leq I_{t h} \leq 3.05$.

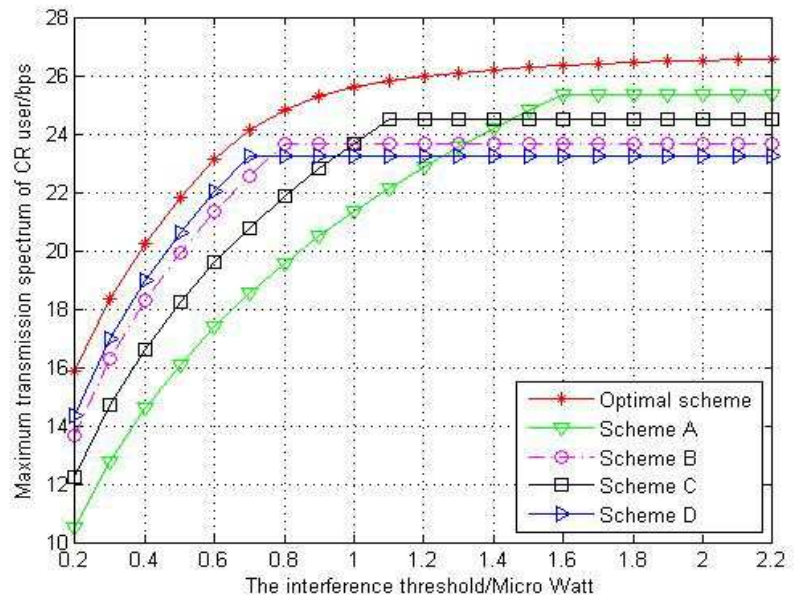

Fig 8. Under different interference threshold of the transmission rate of $\mathrm{CU}$ with $P_{T}=320 \mu \mathrm{W}$.

Figure 8 presents the plan on the relationship between speed ratio and threshold effects in conditions of limited generating capacity.

Similarly, figure 9 presents the plan on the relationship between the ratio of total transmit power and threshold effects in conditions of limited total transmit power $\left(P_{T}=320 \mu \mathrm{W}\right)$.

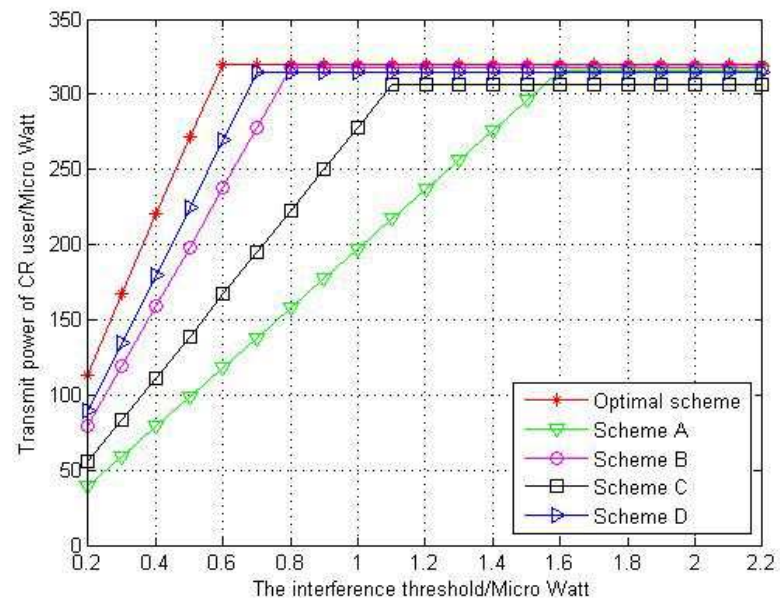

Fig 9. Under different interference threshold of the transmission rate of $C U$ with $P_{T}=320 \mu \mathrm{W}$.

Figure 10 presents optimal method comparison relationship between transmitted capacity limited power and unlimited power up to $10^{3} \mu \mathrm{W}$.

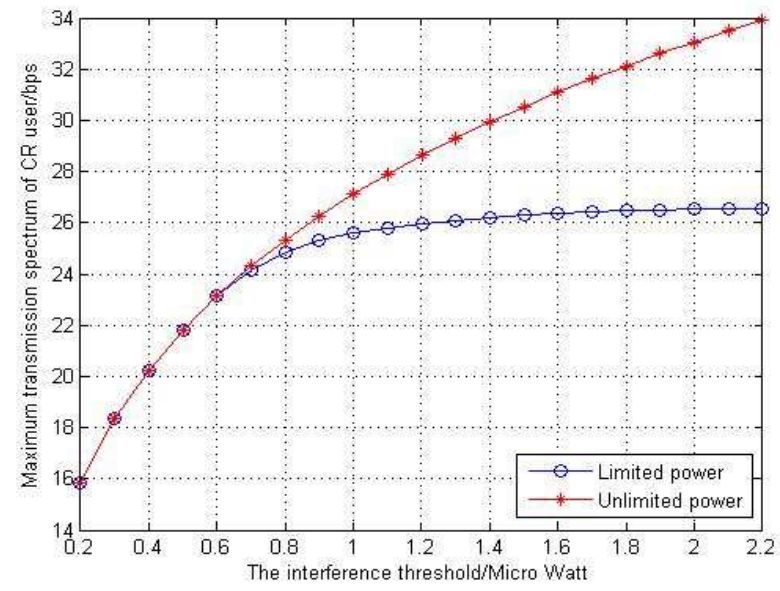

Fig 10. Under different interference threshold of the maximum transmit rate limited power and unlimited power of $C U$.

Figure 11 presents the channel capacity corresponding to different values of BER $=10^{-2}, 10^{-3}, 10^{-4}, 10^{-5}$, and $10^{-6}$. Other parameters are kept the same as above. We can see that the channel capacity increases significantly when the BER requirements are more relaxed. The channel capacity at BER $=$ $10^{-2}$ almost doubles the channel capacity at BER $=10^{-5}$.

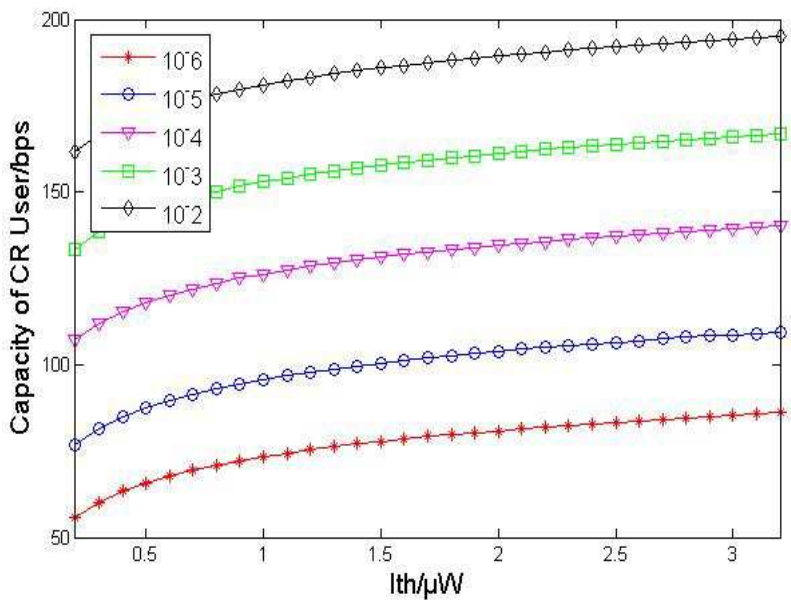

Fig 11. Channel capacity vs. power interference threshold of CUs with different BERs from $10^{-6}$ to $10^{-2}$.

\section{Conclusions}

In this paper, the authors proposed the optimal power allocation problem based on OFDM cognitive radio system. In order to adjust the interference constraint of license users and conduct mathematical modeling by utilizing convex optimization theory, transmitted capacity limited power was compared with unlimited power using Lagrange multiplier method.

The present results prove that the optimization algorithm could maximize the channel transmission rate of $\mathrm{CU}$, when the interference of $\mathrm{CU}$ on LU is in the tolerable scope of LU. In allusion to the complexity and difficultly of optimization scheme, we will study more about the sub-optimization power allocation algorithm and which can be realized easily. 


\section{References}

[1] S. Haykin, Simon, "Cognitive radio: Brain-Empowered Wireless Communications," IEEE Journal on Selected Areas in Communications, vol. 23, pp. 201-220, 2005.

[2] Akyildiz I. F., Won-YeoI Lee, Vuran Mehmet C., Mohanty S., "Next generation, dynamic spectrum access, cognitive radio wireless networks, a survey," Computer Networks of Elsevier, vol. 46, pp. 40-48, April. 2008.

[3] Weiss T. A., Jondral F. K., Spectrum pooling, "An innovative strategy for the enhancement of spectrum efficiency," Communications Magazine of IEEE, vol. 42, pp. S8-14, 2004.

[4] Weiss T., Hillenbrand J., Krohn A., Jondral, F.K, "Mutual interference in OFDM-based spectrum pooling Systems," Proc. IEEE Vehicular Technology Conferenc Spring, IEEE Press, vol. 4, pp. 1873-1877, 2004.

[5] Bansal G., Hossain J., Bhargava V. K., "Optimal and Suboptimal power allocation schemes for OFDM-based cognitive radio systems," IEEE Transactions on Wireless Communications, vol. 7, pp. 4710-4718, 2008.

[6] Wyglinski, Alexander M., "Effects of bit allocation on non-contiguous multicarrier-based cognitive radio transceivers," Proceeding of Vehicular Technology Conference of the IEEE, pp. 1-5, 2006.

[7] G. Bansal, M. J. Hossain, V. K. Bhargava, "A daptive power loading for OFDM-based cognitive radio systems", Proc IEEE ICC, IEEE Press, pp. 5137-5142, 2007.

[8] G. J. Foschini and J. Salz, "Digital communications over fading radio channels," Bell Syst. Tech. J., pp. 429-456, Feb. 1983.

[9] Wang P, Zhao M, Xiao L, "Power allocation in OFDM-based cognitive radio systems", Proc. IEEE Global Communication Conference, IEEE Press, pp. 4061-4065, 2007.

[10] Nguyen Van Vinh, Yang Shouyi and Le Chung Tran, "Power Allocation Algorithm in OFDM-Based Cognitive Radio Systems," Proc. IEEE ComManTel Conf., IEEE Press, pp. 13-18, April 2014. 